\title{
Registros de enfermería sobre el sueño y percepción de los pacientes en una unidad psiquiátrica. Estudio comparativo
}

\author{
Nursing registers on sleep and patient perception \\ in a psychiatric unit. A comparative study
}

\author{
J. Antomás ${ }^{1}$, S. Huarte ${ }^{2}$, C. Gárriz ${ }^{1}$
}

\section{RESUMEN}

Fundamento. El insomnio es un problema frecuente en las personas que sufren enfermedades mentales. El personal de enfermería controla y registra si los pacientes duermen o no.

El objetivo de este trabajo es analizar la concordancia entre las percepciones de los pacientes de una unidad psiquiátrica de agudos sobre la calidad de su sueño y las notas recogidas en el evolutivo de enfermería al respecto.

Material y métodos. Estudio comparativo entre las respuestas dadas por pacientes de nuestra unidad a 126 cuestionarios sobre insomnio en noches concretas y lo reflejado en sus historias de enfermería. Se utilizó una versión reducida de la Escala Atenas de Insomnio (EAI-5)

Resultados. El valor medio obtenido según las respuestas de los pacientes es superior (peor sueño) al dado por las enfermeras, tanto de manera global como por ítem.

La historia de enfermería refleja valores mucho más bajos que los otorgados por el paciente, el análisis de correlación de Pearson proporcionó un coeficiente de 0,26. $\mathrm{El}$ análisis de grado de acuerdo proporcionó un ICC de 0,13 y no difiere de manera significativa al acuerdo nulo.

Conclusiones. Hay discordancia entre las observaciones de enfermería y las sensaciones subjetivas de los pacientes sobre cómo duermen. Refieren dormir peor de lo que refleja la historia de enfermería. La observación externa y la autopercepción no son aspectos antagónicos, sino dos aspectos complementarios del sueño de los pacientes.

Palabras clave. Trastornos del sueño. Registros de enfermería. Servicio de Psiquiatría. Hospital.

\begin{abstract}
Background. Insomnia is a frequent problem in people who suffer from mental illnesses. The nursing staff control and register whether or not patients sleep.

The aim of this article is to analyze the concordance between the perceptions of the patients of a psychiatric unit on the quality of sleep, and the notes in this respect contained in the nursing records.
\end{abstract}

Methods. A comparative study between the answers given by the patients of our unit to 126 questions on insomnia on specific nights, and what was reflected in their nursing records. A reduced version of the Athens Insomnia Scale (AIS-5) was used.

Results. The average value obtained according to the patients' answers was higher (worse sleep) than that given by the nurses, both globally and by item.

The nursing records showed much lower values than those given by the patient; Pearson's correlation analysis gave a coefficient of 0.26 . Analysis of the degree of agreement provided an index of 0.13 that does not differ significantly from nil agreement.

Conclusions. There is a difference between the nursing observations and the subjective sensations of the patients about how they sleep. They indicate that they sleep worse than the nursing records reflect. External observation and self-perception are not antagonistic aspects, but two complementary aspects of patients' sleep.

Key words. Sleep disorders. Nursing records. Psychiatric Department. Hospital.
1. Unidad de Hospitalización Psiquiátrica A. Complejo Hospitalario de Navarra.

2. Centro de Atención a la Mujer de Elizondo. Atención Primaria.

Recepción: 16 de mayo de 2011

Aceptación provisional: 21 de junio de 2011

Aceptación definitiva: 8 de agosto de 2011

\author{
Correspondencia \\ Javier Antomás Osés \\ Pabellón E bajo \\ Complejo Hospitalario de Navarra \\ $\mathrm{C} /$ Irunlarrea, $\mathbf{s} / \mathbf{n}$ \\ 31008 Pamplona \\ E-mail: xabier.antomas.oses@navarra.es
}




\section{INTRODUCCIÓN}

El insomnio es un problema frecuente en las personas que sufren enfermedades mentales $^{1}$. Diferentes textos médicos ${ }^{2,3} \mathrm{y}$ de enfermería ${ }^{4}$ refieren que se debe tener en cuenta la propia percepción subjetiva del paciente a la hora de establecer un diagnóstico y planificar cuidados ${ }^{5,6}$. De hecho, la ICSD-2 (Clasificación Internacional de Trastornos del Sueño) incluye en su catálogo el "Insomnio paradójico", denominado también pseudoinsomnio o mala percepción del sueño. La principal característica es una queja de insomnio grave, sin que pueda objetivarse un trastorno de tal magnitud. Constituye el $5 \%$ de todos los casos de insomnio, y es más frecuente en mujeres ${ }^{7,8}$.

Existen varios métodos para evaluar el insomnio $^{9,10}$. La polisomnografía nocturna, actigrafía de muñeca o el mecanismo de evaluación del sueño ofrecen datos objetivos sobre el sueño de los pacientes. Tienen el problema de requerir aparataje específico y a veces muy costoso y no contemplar la percepción subjetiva de haber dormido o no. Los métodos subjetivos van desde la entrevista clínica a los auto-registros del sueño pasando por multitud de escalas y cuestionarios específicos para diferentes aspectos del sueño. Muchos de estos cuestionarios están estandarizados y validados en varios idiomas ${ }^{11}$. Algunos de los más conocidos enfocados a evaluar los trastornos del sueño en general son el Cuestionario de evaluación del sueño de Leeds ${ }^{12}$, el Índice de calidad del Sueño de Pittsburgh ${ }^{13}$, la Escala de problemas de sueño ${ }^{14} \mathrm{o}$ la Escala Atenas de Insomnio ${ }^{15}$.

Se han realizado diversos estudios que evalúan la fiabilidad de los registros de enfermería sobre el sueño de los pacientes en unidades de cuidados intensivos ${ }^{16-20}$ realizados con control presencial a pie de cama que concluyen que dichos registros son fieles a la realidad. En una unidad de psiquiatría se compararon los registros enfermeros, las percepciones de los pacientes y datos objetivos obtenidos con actigrafía de muñeca, viendo que las observaciones de enfermería se ajustan más a la realidad que las percepciones de los pacientes ${ }^{21}$. En todas estas investigaciones se da la circunstancia que cuando las valoraciones no concuerdan, la del personal de enfermería siempre es más positiva que la del paciente.

La percepción subjetiva del sueño por parte del paciente puede tener incidencia directa sobre su estado de salud general y su actitud ${ }^{22-24}$. Por ello es importante conocer la coherencia existente entre las observaciones de enfermería sobre la calidad del sueño de los pacientes y las percepciones subjetivas de los propios pacientes.

El objetivo de este trabajo es analizar la concordancia entre las percepciones de los pacientes sobre la calidad de su sueño y las interpretaciones de la historia realizadas por parte del personal de enfermería.

\section{MATERIAL Y MÉTODOS}

El estudio se desarrolló en una unidad de psiquiatría para pacientes agudos integrada en la estructura de un hospital terciario. El servicio cuenta con 27 camas de adultos con un índice de ocupación del $94,9 \%$. La plantilla consta de 9 enfermeras y un jefe de unidad. En la citada unidad no se utiliza ningún sistema estandarizado para recoger las percepciones de los pacientes sobre su sueño.

A lo largo de 5 semanas (julio-agosto de 2010) se seleccionaron los pacientes que llevaban más de tres días ingresados, que no habían sido sometidos a contención mecánica o química durante la noche anterior y que estaban en condiciones psicológicas y cognitivas para realizar la prueba. Un total de 47 de 53 pacientes (89\%) aceptaron participar en el estudio. Se les informó del objetivo del estudio, se solicitó el consentimiento informado por escrito y se preservó su confidencialidad.

El instrumento que se utilizó para evaluar el sueño de los pacientes fue la versión reducida ${ }^{25,26}$ de la Escala Atenas de Insomnio (EAI-5) ${ }^{15,27}$. Esta escala es autoaplicable, $\mathrm{y}$ ha sido utilizada en diversos países ${ }^{28} \mathrm{y}$ traducida y validada al español ${ }^{29}$. El periodo de tiempo para el que está diseñada es un mes, pero puede adaptarse a las necesi- 
dades de investigación. La escala completa consta de 8 ítems, los cuatro primeros sobre aspectos cuantitativos del sueño, el quinto sobre la calidad del mismo y los restantes sobre sus repercusiones durante el día. La versión reducida (EAI-5) incluye los 5 primeros ítems (Tabla 1). Cada ítem se responde con una escala tipo Likert que va de 0 "ningún problema" a 3 "problema importante" siendo en total 0 la puntuación mínima y 15 la máxima. Una mayor puntuación indica mayores problemas en el sueño

Tabla 1. Versión reducida de la Athens Insomnia Scale

\begin{tabular}{l} 
INSTRUCCIONES: Esta escala pretende registrar su propia valoración acerca de cualquier dificultad que haya \\
podido experimentar. Por favor, responda los siguientes ítems (marcando con un círculo el número apropiado), \\
indicando las dificultades que le hayan ocurrido la pasada noche. \\
\hline Inducción del sueño (tiempo que tarda en dormirse después de apagar la luz). \\
0. Ningún problema. \\
1. Ligeramente retrasado. \\
2. Marcadamente retrasado. \\
3. Muy retrasado o no durmió. \\
Despertares durante la noche. \\
0. Ningún problema. \\
1. Problema menor. \\
2. Problema considerable. \\
3. Problema serio o no durmió. \\
Despertar final más temprano de lo deseado. \\
1. No fue antes. \\
2. Marcadamente antes. \\
3. Mucho antes o no durmió. \\
0. Suficiente. \\
1. Ligeramente insuficiente. \\
2. Marcadamente insuficiente. \\
3. Muy insuficiente o no durmió. \\
1. Ligeramente insatisfactoria. \\
2. Marcadamente insatisfactoria. \\
\hline Calidad general del sueño (no importa cuánto tiempo durmió). \\
\hline Duración total del sueño.
\end{tabular}

El procedimiento consistió en la realización de una entrevista con el paciente por parte del jefe de la unidad, en la que se obtuvieron los resultados dados por el paciente en cada uno de los ítems de la escala EAI-5 referida al sueño de la noche anterior. Algunos pacientes fueron entrevistados más de un día, por lo que en el pe- 
riodo de estudio se obtuvieron 126 cuestionarios cumplimentados. Adicionalmente y de forma independiente, una enfermera se encargó de valorar e interpretar los 126 historiales de enfermería relativos a esas mismas noches de los pacientes participantes, y puntuó cada ítem de la escala EAI-5 a partir de los mismos. En caso de no haber información explícita sobre alguno de los ítems, se consideró que no había problema relacionado con el mismo. Para evitar un sesgo en la redacción de la historia clínica, las enfermeras del servicio no fueron informadas de los objetivos de la investigación. Además, con el fin de evaluar la variabilidad inter-rater a partir de la información del evolutivo de enfermería, una segunda enfermera externa realizó el mismo análisis a partir de cada historia, puntuando cada uno de los ítems de la escala EAI-5.

\section{Análisis estadístico}

Con el fin de valorar la adecuación de la escala EAI-5 para evaluar el sueño de los pacientes psiquiátricos ingresados en la unidad, se realizó previamente al análisis de grado de acuerdo, un análisis de ítems y de fiabilidad de la escala, así como un análisis factorial exploratorio utilizando las encuestas contestadas por los pacientes por la mañana. Para el análisis de ítems y fiabilidad, se utilizaron estadísticos descriptivos como medias y desviaciones típicas, así como la correlación de Pearson ítem-total medio y correlación entre ítems. Para el estudio de fiabilidad se utilizó el índice alfa de Cronbach con todos los ítems y excluyendo cada ítem. El análisis factorial exploratorio se llevó a cabo mediante análisis de componentes principales, a partir del cual se obtuvo el porcentaje de varianza explicada mediante la extracción de un factor.

Los valores obtenidos a partir de las encuestas contestadas por pacientes y a partir de las valoraciones realizadas según la historia de enfermería se resumieron utilizando estadísticos descriptivos, como la media y la desviación típica, las medianas y rangos intercuartílicos. La dispersión relativa se comparó mediante el coeficiente de variación. Las distribuciones de frecuencia para las puntuaciones totales se representaron mediante un bar-plot conjunto y mediante un quantile-plot con fines comparativos. Para evaluar la correlación entre las puntuaciones totales paciente-enfermera, y para evaluar si uno de los criterios de ellos tiende a dar resultados desviados con respecto a los otros, se utilizó el coeficiente de correlación de Pearson y se estimó la pendiente del análisis de regresión.

Para obtener el grado de acuerdo entre puntuaciones, se utilizó el coeficiente de correlación intraclase (ICC). Para complementar el análisis cuantitativo, se consideró la escala ordinal $(0,1-5$ y $>5)$ analizándose el grado de acuerdo mediante el coeficiente de Kendall ( 0 no acuerdo, 1 acuerdo completo). La misma metodología se utilizó para evaluar el grado de acuerdo entre las dos enfermeras que contestaron al cuestionario a partir de las mismas historias.

\section{RESULTADOS}

\section{Análisis de ítems y fiabilidad}

El análisis de ítems realizado sobre las 126 encuestas contestadas por los pacientes mostró que los valores medios de respuesta para cada ítem están entre 0,45 (ítem 4) y 0,74 (ítem 2), es decir por debajo del punto medio de la escala, y las desviaciones típicas están por debajo de la unidad (entre 0,73 del ítem 1 y 0,83 del ítem 2). El rango de respuesta de todos los ítems es 4 , indicando que todas las opciones de respuesta han sido elegidas.

La fiabilidad de la escala media mediante el valor alfa de Cronbach es 0,85 , indicando alta consistencia interna $(>0,7)$. El valor de correlación ítem-total medio fue de 0,79 y la correlación entre ítems va de 0,35 a 0,76 . La correlación ítem-total supera en todos los casos el valor de $0,30^{30}$ y la eliminación de un ítem no mejora la fiabilidad de la escala. El análisis de componentes principales mostró que el porcentaje de varianza explicada es de $64 \%$.

Los resultados numéricos de las respuestas al cuestionario tanto dadas por los 
pacientes como por las enfermeras a partir la historia de enfermería vienen dados en la Tabla 1, donde se muestran tanto las frecuencias de los valores para cada ítem como los estadísticos descriptivos de las puntuaciones totales.

Tabla 2. Tabla de frecuencias y descriptivos por ítem y para la puntuación total

\begin{tabular}{|c|c|c|c|c|c|c|c|c|c|}
\hline & \multicolumn{6}{|c|}{ Frecuencias por ítem $(\mathrm{n}=126)$} & \multicolumn{3}{|c|}{ Puntación total } \\
\hline & 0 & 1 & 2 & 3 & Media & $\mathrm{DE}$ & Media (DE) ${ }^{(*)}$ & Mediana (RI) ${ }^{(*)}$ & $\mathrm{CV}^{(*)}$ \\
\hline \multicolumn{10}{|c|}{ Puntuación obtenida en la entrevista con el paciente } \\
\hline Ítem 1 & 74 & 40 & 9 & 3 & 0,53 & 0,73 & \multirow[t]{5}{*}{$2,84(3,10)$} & \multirow[t]{5}{*}{$2(1-4)$} & \multirow[t]{5}{*}{1,09} \\
\hline Ítem 2 & 61 & 40 & 22 & 3 & 0,74 & 0,83 & & & \\
\hline Ítem 3 & 66 & 41 & 15 & 4 & 0,66 & 0,81 & & & \\
\hline Ítem 4 & 87 & 23 & 14 & 2 & 0,45 & 0,75 & & & \\
\hline Ítem 5 & 87 & 23 & 13 & 3 & 0,46 & 0,78 & & & \\
\hline
\end{tabular}

Puntuación obtenida a partir de la historia de enfermería según la enfermera de la Unidad

\begin{tabular}{|lcccccc|c|c|c|}
\hline Ítem 1 & 108 & 12 & 6 & 0 & 0,19 & 0,5 \\
\hline Ítem 2 & 111 & 6 & 8 & 1 & 0,2 & 0,58 \\
\hline Ítem 3 & 110 & 14 & 2 & 0 & 0,14 & 0,39 \\
\hline Ítem 4 & 100 & 19 & 7 & 0 & 0,26 & 0,55 & & $0(0-2)$ & \\
\hline Ítem 5 & 83 & 27 & 16 & 0 & 0,47 & 0,71 & & & \\
\hline
\end{tabular}

Puntuación obtenida a partir de la historia de enfermería según la enfermera de Control

\begin{tabular}{|lcccccc|c|c|c|}
\hline Ítem 1 & 115 & 5 & 5 & 1 & 0,14 & 0,5 & $0,94(2,05)$ & $0(0-1)$ & 2,19 \\
\hline Ítem 2 & 104 & 12 & 9 & 1 & 0,26 & 0,62 & & & \\
\hline Ítem 3 & 111 & 9 & 6 & 0 & 0,17 & 0,49 & & & \\
\hline Ítem 4 & 110 & 11 & 4 & 1 & 0,17 & 0,51 & & & \\
\hline Ítem 5 & 111 & 7 & 7 & 1 & 0,19 & 0,56 & & & \\
\hline
\end{tabular}

${ }^{(*)}$ DE: Desviación Estándar; RI: Rango intercuartílico; CV: Coeficiente de Variación

Cabe destacar que el valor medio obtenido según las respuestas de los pacientes es superior al dado por las enfermeras, tanto de manera global como por ítem. En cuanto a la dispersión relativa, observamos que para el paciente la variable está menos dispersa $(\mathrm{CV}=1,09)$, mientras que para la interpretación de la historia de enfermería presenta una mayor dispersión $(\mathrm{CV}=1,54$ y 2,19$)$.

\section{Comparación paciente - historia de enfermería}

En relación a la comparación pacienteenfermera de la unidad, la Fig. 1a) muestra el diagrama de barras conjunto para las puntuaciones totales, e indica que en ambos casos la distribución es asimétrica a la derecha, tomando el cuestionario contestado por la enfermera valores mucho más bajos que los otorgados por el paciente. De hecho, la proporción de cuestionarios en los que el evolutivo de enfermería da un resultado de 0 es de $64 \%$, mientras que la proporción de cuestionarios con resultado global de 0 en pacientes es de $22 \%$. También se observa que ninguna historia de enfermería ha resultado tener una puntuación total mayor a 7, mientras que atendiendo a las respuestas de los pacientes, el $12 \%$ de los cuestionarios tenían una puntuación total superior a este valor. El diagrama de cajas comparativo dado en la Fig. 1b) también indica que hay tendencia a tomar valores más bajos en el cuestionario según el evolutivo de enfermería en mediana. El plot de percentiles se puede 
ver en la Fig. 1c), en el que puntos cerca de la diagonal indicarían que podemos asumir que ambas puntuaciones tienen una misma distribución subyacente. En este caso se observa que los puntos están por debajo de la diagonal, sobre todo en los extremos (por debajo de 4 y por encima de 9), indicando que las puntuaciones de los pacientes son en esos rangos menores a las que los pacientes. Finalmente, el análisis de correlación de Pearson pro- porcionó un coeficiente de 0,26 (95\% IC: $0,09-0,41)$, y es representado mediante el diagrama de dispersión en la Fig. 1d). Se observa que los pares de puntos que relacionan la puntuación de enfermería en relación a los valores de los pacientes no están en la diagonal, siendo la estimación de la pendiente de regresión muy inferior a la unidad, que representaría el ajuste perfecto $(\beta=0,16 ; 95 \%$ IC: $0,05-0,27)$.

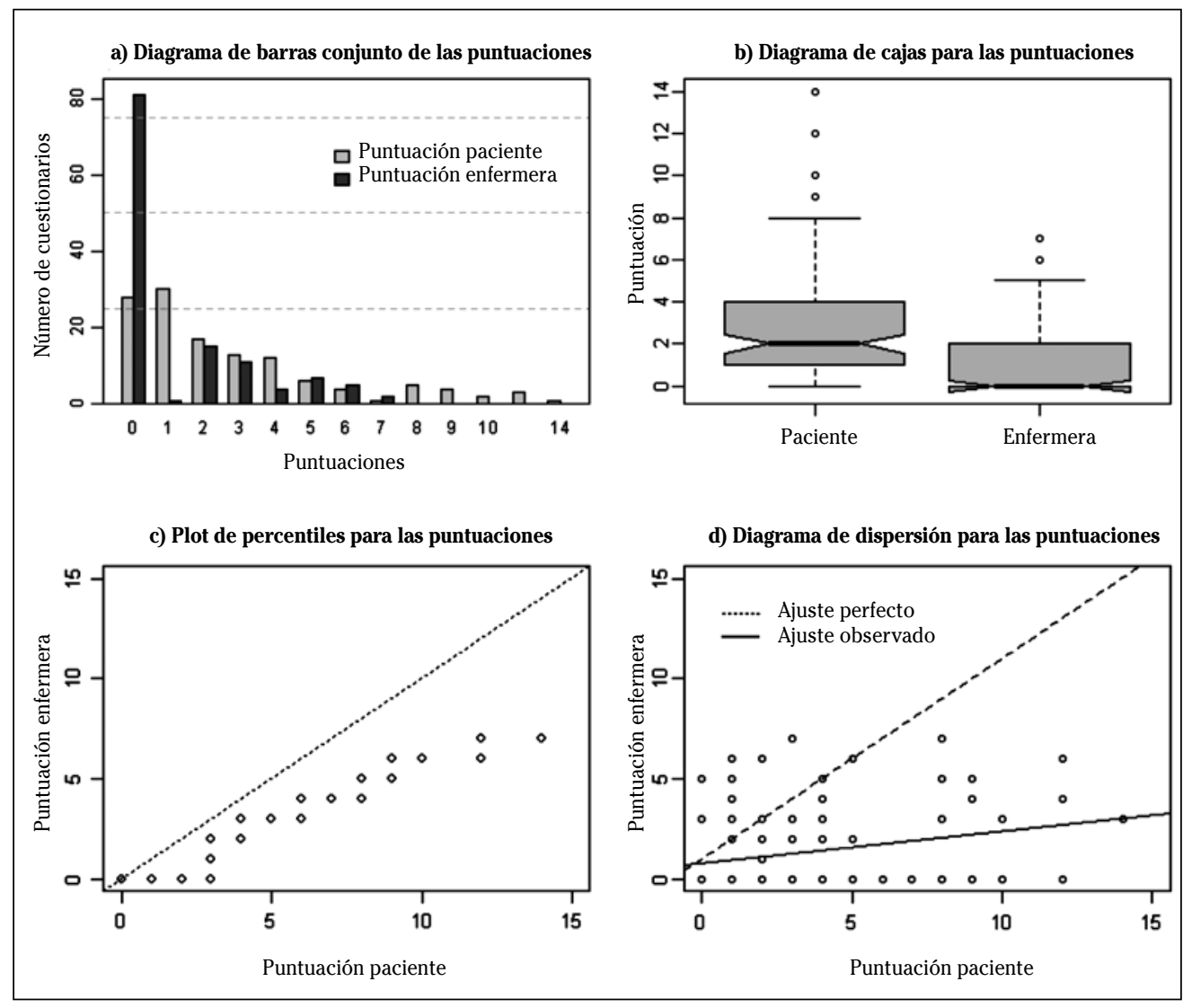

Figura 1. Resultados gráficos de la comparación entre las respuestas dadas por los pacientes y lo obtenido por la enfermera de la unidad tras la lectura de la historia de enfermería.

El análisis de grado de acuerdo proporcionó un ICC de 0,13 (95\% IC; -0,045-0,297). El porcentaje de acuerdo con tolerancia $=1$ (considerando que dos puntuaciones totales son iguales si uno difiere con el otro en como máximo 1 punto) es del $50 \%$. Considerando la variable ordinal de tres categorías para puntuación total $(0,1-5 \mathrm{y}>5)$, el coeficiente de Kendall entre pacienteenfermera fue $\mathrm{Wt}=0,625 ; \mathrm{p}=0,030$. Finalmen- 
te, si consideramos los resultados para cada ítem, observamos que el coeficiente de concordancia de Kendall fue Wt $=0,578$ $(\mathrm{p}=0,113)$ para el ítem $1, \mathrm{Wt}=0,576(\mathrm{p}=0,119)$ para el ítem $2, \mathrm{Wt}=0,509(\mathrm{p}=0,426)$ para el ítem $3, \mathrm{Wt}=0,511(\mathrm{p}=0,415)$ para el ítem $4 \mathrm{y}$ $\mathrm{Wt}=0,584(\mathrm{p}=0,096)$ para el ítem 5 . Todos ellos indican que el grado de acuerdo no difiere de manera significativa al acuerdo nulo.

Respecto a la comparación entre las valoraciones de las historias de enfermería, el análisis de correlación de Pearson proporcionó un coeficiente de 0,80 (95\% IC: $0,73-0,86)$. Se obtuvo un ICC de 0,79 (95\% IC; $0,71-0,85)$. El porcentaje de acuerdo con tolerancia $=1$ es del $81 \%$. Considerando la variable ordinal de tres categorías (0, 1-5 y $>5$ ), el coeficiente de Kendall fue $\mathrm{Wt}=0,953$, $\mathrm{p}<0,01$. Finalmente, si consideramos los resultados para cada ítem, observamos que el coeficiente de concordancia de Kendall fue $\mathrm{Wt}=0,872(\mathrm{p}<0,001)$ para el ítem 1 , $\mathrm{Wt}=0,749(\mathrm{p}<0.001)$ para el ítem $2, \mathrm{Wt}=0,860$ $(\mathrm{p}<0,001)$ para el ítem $3, \mathrm{Wt}=0,765(\mathrm{p}<0,001)$ para el ítem 4 y Wt=0,775 $(\mathrm{p}<0,001)$ para el ítem 5. Todos ellos indican que el grado de acuerdo difiere del no acuerdo, siendo más alto el acuerdo para los ítems 1 y 3 que para el resto.

\section{DISCUSIÓN}

Este estudio muestra que hay una clara discordancia entre las observaciones de enfermería y las sensaciones subjetivas expresadas por los pacientes sobre cómo duermen.

Para estar seguros de que en la interpretación de la historia de enfermería no había variabilidad inter-enfermeras, ésta se realizó de manera independiente por dos enfermeras, resultando un alto grado de acuerdo entre ambas.

Comparando las puntuaciones otorgadas por los pacientes con las de la enfermera del servicio, se aprecia a primera vista una diferencia sustancial (ICC $=0,13$ y $\mathrm{Wt}=0,625)$, siendo los valores de los pacientes mucho más altos en general. De manera que refieren sentir una peor calidad del sueño de lo que refleja la historia de enfermería.

Si se analizan individualmente cada uno de los ítems la concordancia es muy baja, especialmente en los ítems 3 y 4 de la escala $(\mathrm{Wt}=0,509$ y 0,511). Una explicación plausible para este dato podría ser que el horario "obligado" de sueño en la unidad es de 24:00 a 8:00. Por ello, los ítems "duración total del sueño" y "despertar antes de lo deseado" pueden ser considerados adecuados por parte de enfermería si se ajustan a él. Sin embargo, para muchos pacientes tener que levantarse a las 8:00 puede parecerles excesivamente temprano e interpretar que no duermen tiempo suficiente.

Anteriormente Krahn concluyó que los pacientes psiquiátricos tienden a subestimar la calidad del sueño una vez comparada con datos objetivos y que las observaciones de enfermería se ajustan más a la realidad ${ }^{21}$. Otros estudios muestran que las personas que duermen mal tienden a infravalorar su sueño ${ }^{31,32}$. En nuestro caso se ha observado por parte del entrevistador cómo algunos pacientes tienden a dar valores negativos en la escala como forma de canalizar su enfado o frustración por su situación en el momento de la cumplimentación, verbalizándolo expresamente. Por todo ello creemos que no se deben plantear la observación externa y la autopercepción como aspectos antagónicos del sueño de los pacientes, sino más bien como dos facetas de una misma realidad; una parte de informaciones objetivas y otra de percepciones subjetivas que se complementan y enriquecen mutuamente. No obstante lo dicho, la historia de enfermería es una fuente de datos fundamental en el seguimiento de la evolución clínica y se debe trabajar en la mejora del registro de las observaciones.

Para prestar una atención de calidad en unidades de psiquiatría es importante la detección de problemas de sueño, objetivos o subjetivos, ya que van a tener repercusión en el funcionamiento diurno de los pacientes.

Los cuidados que el equipo de enfermería planifica y presta se verán influidos por la actitud y receptividad de sus desti- 
natarios y éstas no van a ser buenas si el descanso nocturno no es adecuado. Asimismo en esa planificación van a incluirse en caso necesario medidas de higiene del sueño como primer paso para evitar estos problemas y si fuera necesario se puede plantear un abordaje farmacológico con el equipo médico.

Opinamos que la mera toma de conciencia por parte del equipo de enfermería de la existencia de las discordancias objetivadas en este estudio redundará positivamente tanto en el control nocturno de los pacientes como en la observación de las repercusiones del insomnio durante el día. No obstante, se hace necesario a nuestro entender la introducción de una escala de valoración del sueño entre los registros de enfermería.

Respecto a la EAI, se ha podido ver que es fiable y con una buena consistencia interna en la línea de estudios anteriores de validación de la misma. Esto nos anima a continuar usándola en posteriores estudios o intervenciones en nuestra unidad en conjunción con las observaciones enfermeras.

A la vista de los resultados se abren diversas opciones de intervención e investigación. Por un lado, la realización de estudios epidemiológicos sobre el insomnio en las unidades de hospitalización de agudos para comprobar su prevalencia así como definir los perfiles psicopatológicos con mayor incidencia. De esta manera se podrán diseñar actuaciones específicas para grupos concretos de pacientes según sus patologías. También se baraja la introducción de la EAI de manera general en los registros de nuestra unidad para detectar casos concretos y la ejecución de planes de actividades personalizables de higiene del sueño para ellos.

\section{Agradecimientos}

Los autores quieren agradecer su inestimable ayuda en aspectos metodológicos y estadísticos a Berta Ibáñez Beroiz y Koldo Cambra Contín de la Fundación Miguel Servet.

\section{BIBLIOGRAFÍA}

1. Sarrais F, de Castro Manglano P. El insomnio. An Sist Sanit Navar 2007; 30 (Supl 1) 121-134.

2. World Health Organization. International statistical classification of diseases and related health problems. 10th revision, 2nd edition. ed. Geneva: World Health Organization 2004.

3. American Psychiatric Association, American Psychiatric Association. Task Force on DSMIV. Diagnostic and statistical manual of mental disorders: DSM-IV-TR. 4th ed. Washington, DC: American Psychiatric Association 2000.

4. North American Nursing Diagnosis Association, NANDA International. Diagnósticos enfermeros: definiciones y clasificación (20072008). 2008 ed. Madrid: Elsevier España 2008.

5. Moorhead S, Johnson M, Meridean LM, Swanson E. Clasificación de resultados de enfermería (NOC). 4 ed. Barcelona: Elsevier España 2009.

6. BulecheK GM, Dochterman JM. Clasificación de intervenciones de enfermería (NIC). 5 ed. Barelona: Elsevier España 2009.

7. Gallego Perez-Larraya J, Toledo JB, Urrestarazu E, IRIARTE J. Clasificación de los trastornos del sueño. An Sist Sanit Navar 2007; 30 Suppl 1: 19-36.

8. American Academy of Sleep Medicine. The international classification of sleep disorders: diagnostic and coding manual. 2nd ed. Westchester, Ill.: American Academy of Sleep Medicine 2005.

9. Garcia dE GuRtubay I. Estudios diagnósticos en patología del sueño. An Sist Sanit Navar 2007; 30 (Supl 1) 37-51.

10. RuIz C. Revisión de los diversos métodos de evaluación del trastorno de insomnio. Anal Psicología 2007; 23: 109-117.

11. Lomeli HA, Perez-Olmos I, Talero-Gutierrez C, Moreno CB, Gonzalez-Reyes R, Palacios L et al. Sleep evaluation scales and questionaries: a review. Actas Esp Psiquiatr 2008; 36: 50-59.

12. Parrott AC, Hindmarch I. The leeds sleep evaluation questionnaire in psychopharmacological investigations - a review. Psychopharmacology (Berl) 1980; 71: 173-179.

13. Buysse DJ, Reynolds CF 3rd, Monk TH, Berman SR, KupFer DJ. The Pittsburgh Sleep Quality Index: a new instrument for psychiatric practice and research. Psychiatry Res 1989; 28: 193-213. 
14. Jenkins CD, Stanton BA, Niemcryk SJ, Rose RM. A scale for the estimation of sleep problems in clinical research. J Clin Epidemiol 1988; 41:313-321.

15. Soldatos CR, Dikeos DG, Paparrigopoulos TJ. Athens Insomnia Scale: validation of an instrument based on ICD-10 criteria. J Psychosom Res 2000; 48: 555-560.

16. Bourne RS, Minelli C, Mills GH, Kandler R. Clinical review: Sleep measurement in critical care patients: research and clinical implications. Crit Care 2007; 11: 226.

17. Edell-Gustafsson U, Aren C, Hamrin E, Hetta J. Nurses' notes on sleep patterns in patients undergoing coronary artery bypass surgery: a retrospective evaluation of patient records. J Adv Nurs 1994; 20: 331-336.

18. Edwards GB, Schuring LM. Pilot study: validating staff nurses' observations of sleep and wake states among critically ill patients, using polysomnography. Am J Crit Care 1993; 2 : 125-131.

19. Nicolas A, Aizpitarte E, Iruarrizaga A, Vazquez M, Margall A, Asiain C. Perception of night-time sleep by surgical patients in an intensive care unit. Nurs Crit Care 2008; 13: 25-33.

20. Richardson A, Crow W, Coghill E, Turnock C. A comparison of sleep assessment tools by nurses and patients in critical care. J Clin Nurs 2007; 16: 1660-1668.

21. Krahn LE, Lin SC, Wisbey J, Rummans TA, O'CONNOR MK. Assessing sleep in psychiatric inpatients: nurse and patient reports versus wrist actigraphy. Ann Clin Psychiatry 1997; 9: 203-210.

22. Suzuki M, Konno C, Furihata R, Osaki K, Uchiyama M. [Insomnia associated with psychiatric disorders]. Nippon Rinsho 2009; 67: 1507-1512.
23. Li SX, Lam SP, Yu MW, Zhang J, Wing YK. Nocturnal sleep disturbances as a predictor of suicide attempts among psychiatric outpatients: a clinical, epidemiologic, prospective study. J Clin Psychiatry 2010; 71: 14401446.

24. Aвad VC, Guilleminault C. Sleep and psychiatry. Dialogues Clin Neurosci 2005; 7: 291-303.

25. Sierra JC, Santos Iglesias P, Martínez Alonso A. Análisis de la fiabilidad y validez de la versión reducida en español de la Athens Insomnia Scale (EAI-5). C Med Psicosom 2008; 88: 11-16.

26. Gullera G, Gómez J, Ruiz C. La versión reducida en español de la Athens Insomnia Scale 2006 [cited 2010 14/2/2010].

27. Soldatos CR, Dikeos DG, Paparrigopoulos TJ. The diagnostic validity of the Athens Insomnia Scale. J Psychosom Res 2003; 55: 263-267.

28. Soldatos CR, Allaert FA, Ohta T, Dikeos DG. How do individuals sleep around the world? Results from a single-day survey in ten countries. Sleep Med 2005; 6: 5-13.

29. Nanclares Portocarrero A, Jiménez-Genchi A. Estudio de validación de la traducción al español de la escala Atenas de Insomnio. Salud Mental 2005; 28: 34-39.

30. Nunnally JC, Bernstein IJ. Teoría psicométrica. 3 ed. México, D.F.: McGraw-Hill 1995.

31. Shaver JL, Giblin E, Paulsen V. Sleep quality subtypes in midlife women. Sleep 1991; 14 : 18-23.

32. Coates TJ, Killen JD, George J, Marchini E, SilVERMAN S, Thoresen C. Estimating sleep parameters: a multitrait-multimethod analysis. J Consult Clin Psychol 1982; 50: 345-352. 